\title{
Einfluss eines komplementärmedizinischen telefonischen Beratungssystems auf den Antibiotikaeinsatz bei Nutztieren in der Schweiz
}

\author{
Ariane Maeschli ${ }^{\mathrm{a}}$ Andreas Schmidt ${ }^{\mathrm{b}}$ Werner Ammann ${ }^{\mathrm{c}}$ \\ Priska Schurtenberger $^{d}$ Erika Maurer ${ }^{\mathrm{e}}$ Michael Walkenhorst ${ }^{\mathrm{a}}$

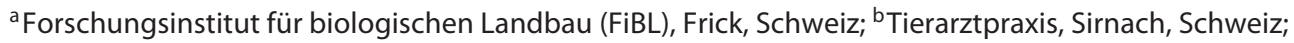

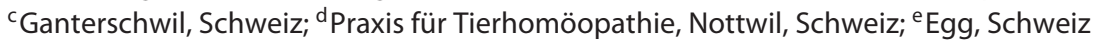

\section{Schlüsselwörter \\ Antibiotikareduktion · Komplementärmedizin . \\ Nutztiergesundheit · Beratungsprogramm . \\ Behandlungsinzidenz}

\begin{abstract}
Zusammenfassung
Hintergrund: Der Antibiotikaeinsatz bei Nutztieren fördert die Entwicklung antibiotikaresistenter Bakterien. Die Komplementärmedizin könnte einen Beitrag zur Reduktion von Antibiotika leisten, wird bisher jedoch nicht flächendeckend angeboten. Das Beratungsprogramm "Kometian" unterstützt teilnehmende Landwirte darin, ihre Tiere komplementärmedizinisch zu behandeln. Methoden und Resultate: 128 von 223 teilnehmenden Betrieben nutzten in den ersten 3 Projektjahren die telefonische Beratung von Kometian. Die Mehrheit der Landwirte hielt Milchkühe. Frühestens 1 Woche nach der Kometianberatung wurde eine telefonische NachfragebeimTierbesitzerhinsichtlich desGesundheitszustandes des Tieres durchgeführt. Es konnten so 661 Beratungsfälle recherchiert werden. In 486 Fällen erfolgte keine weitere schulmedizinische Behandlung, in 373 Fällen gab der Landwirt einen Behandlungserfolg an. Von 188 angefragten und seit mindestens einem Jahr an Kometian teilnehmenden Betrieben stellten 46 ihre Behandlungsjournale zur Verfügung. Die betriebliche antibiotische Behandlungsinzidenz sank signifikant $(p<0,001)$ von 27 vor Beitritt auf 18 Behandlungen pro 100 Tiere im ersten Jahr. Schlussfolgerung: Es scheint, dass Kometian einen Beitrag dazu leisten konnte, den Antibiotikaeinsatz auf Betriebsebene zu reduzieren.
\end{abstract}

(c) 2019 S. Karger AG, Basel
(9) 2019 S. Karger AG, Basel

\author{
Influence of a Complementary and Alternative \\ Medical Advisory Program by Telephone on the Use \\ of Antibiotics in Livestock in Switzerland
}

\section{Keywords}

Reduction of antibiotics - Complementary and alternative medicine - Livestock health · Advisory program · Treatment incidence

\begin{abstract}
Background: A regular use of antibiotics in farm animals is assumed to induce antibiotic-resistant bacteria. Complementary and alternative medicine (CAM) may contribute to a reduction of antibiotic use but it is not extensively offered by Swiss veterinarians. The advisory program "Kometian" supports farmers in treating their animals with CAM. Methods and Results: 128 out of 223 participating farmers used the telephone consultations of Kometian in the first 3 years of the project. The majority of the farmers kept dairy cattle. No sooner than 1 week after such a consultation, the famer was questioned by phone about the health progress of the animal. Thus, the development of health could be verified in 661 cases (of 714). 486 cases were not treated further by conventional medicine; in 373 cases the farmer reported a positive treatment effect. Of 188 examined farms, which had participated in the advisory program for at least 1 year, 46 made their treatment records available. The incidence of antibi-
\end{abstract}


otic treatment decreased significantly $(p<0.001)$ from 27 before entering the program to 18 treatments per 100 animals in the first year. Conclusion: It seems that the advisory program Kometian could contribute to reduce the use of antibiotics on the farm level.

\section{Einleitung}

In der Schweiz werden für landwirtschaftliche Nutztiere rund 38 Tonnen antibiotische Wirkstoffe eingesetzt [1]. Ein regelmässiger Antibiotikaeinsatz kann das Risiko zur Entwicklung resistenter Bakterien im Tier, aber auch in der Umwelt erhöhen [2, 3].

Als wichtigste Massnahme zur Reduktion von Antibiotika auf dem landwirtschaftlichen Betrieb erweist sich die Prävention. Verschiedene Studien zeigen, dass ein optimales Management die Voraussetzung für eine gute oder verbesserte Tiergesundheit bei gleichzeitiger Reduktion von Antibiotika ist [4-6]. Jedoch können Erkrankungen von Einzeltieren auch bei optimalem Management auftreten.

Seitens der schweizerischen Landwirte und Landwirtinnen besteht eine erhebliche Nachfrage, sowohl im Bereich der biologischen als auch in der integriert produzierenden Landwirtschaft, vorzugsweise komplementärmedizinische Therapiemethoden einzusetzen. Aufgrund der geringen Dichte komplementärmedizinisch weitergebildeter Tierärztinnen und Tierärzte besteht eine erhebliche Diskrepanz zwischen Angebot und Nachfrage, obwohl die seit 2009 in der schweizerischen Bundesverfassung verankerte Komplementärmedizin [7] nicht auf die Humanmedizin begrenzt ist. Daher behandeln Landwirte in der Schweiz und in anderen Ländern Europas ihre Nutztiere selbstständig mit pflanzlichen Hausmitteln [8-10] oder mit Homöopathie $[11,12]$.

Anfang 2012 wurde das Projekt "Kometian" initiiert [13], welches eine telefonische komplementärmedizinische Beratung betreibt. Über ein Dutzend vorwiegend homöopathisch versierte Tierärzte [14] und Tierheilpraktiker, letztere mit von den beiden schweizerischen Berufsverbänden anerkannter Ausbildung (Tierheilpraktikerverband [15] oder Homöopathieverband Schweiz [16]) stehen ratsuchenden Landwirten seither telefonisch rund um die Uhr zur Verfügung. Bei Bedarf werden auch Betriebsbesuche durchgeführt.

Mit der vorliegenden Studie sollte abgeklärt werden, (a) wie die Landwirte die gesundheitliche Entwicklung einzelner Tiere in Folge der von Kometian vorgeschlagenen therapeutischen Massnahmen einschätzten und (b) ob die Betriebe im Verlauf ihres ersten Mitgliedschaftsjahres bei Kometian ihre antibiotische Behandlungsinzidenz verändert haben.

\section{Material und Methoden}

Nach 3 Projektjahren, im Dezember 2015, waren 223 Betriebe Mitglied von Kometian, davon 139 Bio-Betriebe. 216 der 223 Betriebe gaben in der Anfangsbefragung im Rahmen der Anmeldung an, den Einsatz von Antibiotika reduzieren zu wollen. Fast alle (206 Betriebe) gaben darüber hinaus an, bereits vor Beitritt zu Kometian bei ihren Tieren Komplementärmedizin eingesetzt zu haben. Ein Grossteil der Betriebe hielt Milchvieh, aber auch Mutterkuh- und Schweinehalter waren zahlreich vertreten. Insgesamt hielten die Betriebe zusammen rund 4'400 Milchkühe, 700 Mutterkühe, 1'800 Aufzuchtrinder, 800 Mastrinder, 900 Mastkälber, 1'700 Muttersauen, 4'800 Mastschweine, 200 Milchschafe, 1'500 Fleischschafe, 400 Ziegen und 200 Pferde. Bis Ende Mai 2015 hatten 128 der 223 Betriebe die Beratung von Kometian mindestens einmal genutzt.

$\mathrm{Zu}$ Projekteintritt wurden die Landwirte instruiert, auf welche Symptome (inklusive spezifisch für homöopathische Diagnostik wichtige Symptome) sie im Krankheitsfall zu achten hatten, um dem Berater am Telefon ein möglichst genaues Bild des Tieres zu übermitteln.

\section{Einzeltierberatung - gesundheitliche Entwicklung der}

beratenen Fälle

Bei der Einzeltierberatung per Telefon, die ausschliesslich auf Eigeninitiative des ratsuchenden Landwirtes stattfand, wurde dem Kometianberater die Symptomatik des aktuellen Problems geschildert. Basierend auf diesem Vorbericht und eigener Nachfragen gab der Berater Empfehlungen zur homöopathischen oder einer anderen komplementärmedizinischen Behandlung, sowie zu weiteren tierindividuellen Massnahmen (Management, Fütterung) ab. Eine gute Tierbeobachtung seitens des Landwirtes war zur optimalen Beratung Voraussetzung. Im Beratungsgespräch schätzte der Kometianberater ab, ob aus tierschützerischen Gründen unmittelbar der Hoftierarzt miteinbezogen werden sollte. Zum Teil wurde, nicht selten in Absprache mit dem Hoftierarzt, die Kometianberatung auch als letzte Instanz in Anspruch genommen, bei Tieren, die aus Sicht des Hoftierarztes als austherapiert angesehen wurden. Die letzte Entscheidung, ob und mit welcher Methode ein Tier therapiert wurde, lag beim Landwirt.

Der Kometianberater dokumentierte jeden Fall nach internen Vorgaben. Diese Dokumentation wurde von einer zweiten Fachperson zur internen Qualitätssicherung überprüft. Für jeden Fall wurde festgehalten, bis zu welchem Datum das Tier, nach Meinung des Beraters, eine deutliche Besserung zeigen sollte. Frühestens zu diesem Zeitpunkt wurde, im Rahmen der externen Qualitätssicherung und der wissenschaftlichen Begleitung des Projektes, eine telefonische Nachfrage beim Landwirt hinsichtlich der gesundheitlichen Entwicklung des Einzeltieres durchgeführt.

Bei dieser telefonischen Befragung gaben die Landwirte Auskunft über die Umsetzung der Beratungsempfehlung, den Krankheitsverlauf, zusätzliche Behandlungen (differenziert nach schulmedizinisch oder komplementärmedizinisch), welche zwischen dem Datum der Beratung und der Nachfrage lagen und über den aktuellen Gesundheitszustand bzw. den Verbleib des Tieres. Der Gesundheitszustand wurde in "Behandlungserfolg", "kein Behandlungserfolg" und "tot" unterteilt. Als Behandlungserfolg galt eine vollständige Ausheilung aus Sicht des Landwirts sowie das Erreichen eines guten Allgemeinzustandes mit weiterer Nutzung für die landwirtschaftliche Produktion (z.B. klinische Ausheilung einer Mastitis mit verbleibender Erhöhung der Zellzahlen, Ausheilung einer Metritis, jedoch verzögerter Zykluseintritt usw.). "Kein Behandlungserfolg" bedeutete, dass das Tier ohne die erhoffte Verbesserung des Gesundheitszustandes noch genutzt wurde, da das Tierwohl nicht oder kaum beeinträchtigt war (z.B. chronische 
Tabelle 1. Anzahl Bestände pro Tierkategorie von den 188 Betrieben, welche angefragt wurden das Behandlungsjournal zur Verfügung zu stellen (Mehrfachnennungen pro Betrieb möglich)

\begin{tabular}{|c|c|c|c|c|c|c|c|c|c|}
\hline \multirow{2}{*}{$\begin{array}{l}\text { Anzahl Bestände: } \\
\text { Tierkategorie: }\end{array}$} & \multicolumn{2}{|c|}{$\begin{array}{l}\text { Adulte Rinder } \\
168\end{array}$} & \multicolumn{3}{|c|}{$\begin{array}{l}\text { Jungrinder } \\
145\end{array}$} & \multicolumn{2}{|l|}{$\begin{array}{l}\text { Schweine } \\
62\end{array}$} & \multicolumn{2}{|c|}{$\begin{array}{l}\text { Kleinwiederkäuer } \\
59\end{array}$} \\
\hline & $\begin{array}{l}\text { Milch- } \\
\text { kuh }\end{array}$ & $\begin{array}{l}\text { Mutter- } \\
\text { kuh }\end{array}$ & $\begin{array}{l}\text { Mast- } \\
\text { kalb }\end{array}$ & $\begin{array}{l}\text { Mast- } \\
\text { rind }\end{array}$ & $\begin{array}{l}\text { Aufzucht- } \\
\text { rind }\end{array}$ & Muttersau & $\begin{array}{l}\text { Mast- } \\
\text { schwein }\end{array}$ & Schaf & Ziege \\
\hline Anzahl Bestände & 138 & 37 & 74 & 32 & 122 & 30 & 53 & 33 & 32 \\
\hline $\begin{array}{l}\text { Anzahl Tiere/Bestand } \\
\text { MW (min.-max.) }\end{array}$ & $\begin{array}{l}27,1 \\
(1-90)\end{array}$ & $\begin{array}{l}14,5 \\
(1-50)\end{array}$ & $\begin{array}{l}10,1 \\
(1-100)\end{array}$ & $\begin{array}{l}20,2 \\
(1-160)\end{array}$ & $\begin{array}{l}12,5 \\
(1-60)\end{array}$ & $\begin{array}{l}49,1 \\
(1-380)\end{array}$ & $\begin{array}{l}83,9 \\
(2-500)\end{array}$ & $\begin{array}{l}43,6 \\
(3-500)\end{array}$ & $\begin{array}{l}7,8 \\
(1-60)\end{array}$ \\
\hline
\end{tabular}

Tabelle 2. Anzahl Bestände pro Tierkategorie von den 46 Betrieben, von welchen das Behandlungsjournal ausgewertet wurde (Mehrfachnennungen pro Betrieb möglich)

\begin{tabular}{|c|c|c|c|c|c|c|c|c|c|}
\hline \multirow{2}{*}{$\begin{array}{l}\text { Anzahl Bestände: } \\
\text { Tierkategorie: }\end{array}$} & \multicolumn{2}{|c|}{$\begin{array}{l}\text { Adulte Rinder } \\
44\end{array}$} & \multicolumn{3}{|c|}{$\begin{array}{l}\text { Jungrinder* } \\
40\end{array}$} & \multicolumn{2}{|l|}{$\begin{array}{l}\text { Schwein* } \\
13\end{array}$} & \multicolumn{2}{|c|}{$\begin{array}{l}\text { Kleinwiederkäuer* } \\
16\end{array}$} \\
\hline & Milchkuh* & Mutterkuh* & $\begin{array}{l}\text { Mast- } \\
\text { kalb }\end{array}$ & $\begin{array}{l}\text { Mast- } \\
\text { rind }\end{array}$ & $\begin{array}{l}\text { Aufzucht- } \\
\text { rind }\end{array}$ & Muttersau & $\begin{array}{l}\text { Mast- } \\
\text { schwein }\end{array}$ & Schaf & Ziege \\
\hline Anzahl Bestände & 28 & 16 & 18 & 11 & 30 & 6 & 11 & 10 & 7 \\
\hline $\begin{array}{l}\text { Anzahl Tiere/Bestand } \\
\text { MW (min.-max.) }\end{array}$ & $\begin{array}{l}26,0 \\
(10-50)\end{array}$ & $\begin{array}{l}15,7 \\
(3-25)\end{array}$ & $\begin{array}{l}10,0 \\
(1-25)\end{array}$ & $\begin{array}{l}9,6 \\
(1-30)\end{array}$ & $\begin{array}{l}13,1 \\
(1-35)\end{array}$ & $\begin{array}{l}13,2 \\
(2-30)\end{array}$ & $\begin{array}{l}20,2 \\
(2-150)\end{array}$ & $\begin{array}{l}27,7 \\
(6-120)\end{array}$ & $\begin{array}{l}13,4 \\
(2-60)\end{array}$ \\
\hline
\end{tabular}

* Die Behandlungsinzidenz dieser Bestände bzw. Tierkategorien wurde separat analysiert.

Mastitis, Fruchtbarkeitsprobleme, chronische Erkrankungen der Gliedmassen) oder dass solche Tiere der regulären Schlachtung zugeführt wurden. Schliesslich bedeutete "tot", dass das Tier aufgrund der Schwere der Erkrankung euthanasiert oder notgeschlachtet werden musste oder aber verendet war. Die Ergebnisse dieser Befragungen wurden deskriptiv ausgewertet.

\section{Entwicklung des betrieblichen Antibiotikaeinsatzes}

Im Mai 2015 wurden 188 Landwirte, welche zu diesem Zeitpunkt seit mindestens einem Jahr bei Kometian angemeldet waren, angefragt, ihre Behandlungsaufzeichnungen vom 1. Januar 2011 bis 31. Dezember 2014 zur Verfügung zu stellen. Die meisten Betriebe hielten Milchvieh (161 der 188 Betriebe; Tab. 1). Die Rücklaufquote betrug 27\% (51 Behandlungsjournale). Von 5 Betrieben waren die Behandlungsjournale nicht vollständig oder es fehlten Angaben zum Betrieb. Für die vorliegende Untersuchung konnten so die antibiotischen Behandlungen von 46 Betrieben (25\%) ausgewertet werden (Tab. 2). Für die Auswertungen wurden die antibiotischen Behandlungen (durchschnittliche Anzahl antibiotischer Behandlungen pro Tier und Jahr auf Ebene des Betriebes) im Jahr 0 zu Jahr 1 verglichen. Mehrere Medikamentengaben beim selben Tier zum selben Behandlungsgrund zählten dabei als eine Behandlung, wenn keine Behandlungsunterbrechung von $>7$ Tagen vorlag [6]. Die Behandlungen wurden jeweils einer Tierkategorie zugeordnet (Tab. 2). Die Behandlung eines Wurfes Saugferkel wurde als eine Behandlung der Muttersau gezählt.

Für die statistischen Auswertungen wurden Zucht- und Mastschweine als "Schweine" sowie Ziegen und Schafe als "Kleinwiederkäuer" zusammengefasst. Um die betrieblichen Veränderungen der antibiotischen Behandlungsinzidenz von Jahr 0 zu Jahr 1 vergleichen zu können, wurden die beiden Projektjahre eines Betriebs als abhängige Variablen betrachtet. Da es sich beim Antibiotikaeinsatz um eine nicht normal verteilte Variable handelt, wurde die Analyse mit dem Wilcoxon-signed-rank-Test erstellt.
Als Signifikanzniveau wurde $\alpha \leq 0,05$ festgelegt. Für die statistischen Auswertungen wurde das Programm SPSS (Version 20) verwendet.

\section{Ergebnisse}

\section{Einzeltierberatung - gesundheitliche Entwicklung der} beratenen Fälle

In den ersten 3 Jahren des Projektes Kometian wurde zwischen dem 1. Februar 2012 und dem 31. März 2015 der Krankheitsverlauf aus Sicht des Landwirtes von 661 der insgesamt 714 von Kometian beratenen Fällen erhoben. Von den 53 nicht erhobenen Fällen konnte in 35 Fällen der Landwirt nach 4 Anrufen nicht erreicht werden. In 18 Fällen konnte der Krankheitsverlauf nicht beurteilt werden.

Der grösste Teil der 661 telefonisch nachkontrollierten Fälle fiel auf Kühe und Rinder im Alter von mehr als 6 Monaten. Mit knapp einem Drittel aller Anfragen (32\%) stellten Mastitiden den grössten Anteil aller Beratungsanfragen dar (Abb. 1).

486 Fälle (74\%) wurden nicht schulmedizinisch nachbehandelt. In 373 Fällen gab der Landwirt einen Behandlungserfolg an (Abb. 2). Bei den übrigen 113 Fällen handelte es sich in der Regel um chronische Fälle (insbesondere um chronisch-subklinische Mastitiden oder Fruchtbarkeitsprobleme) oder sehr schwere Fälle, welche 


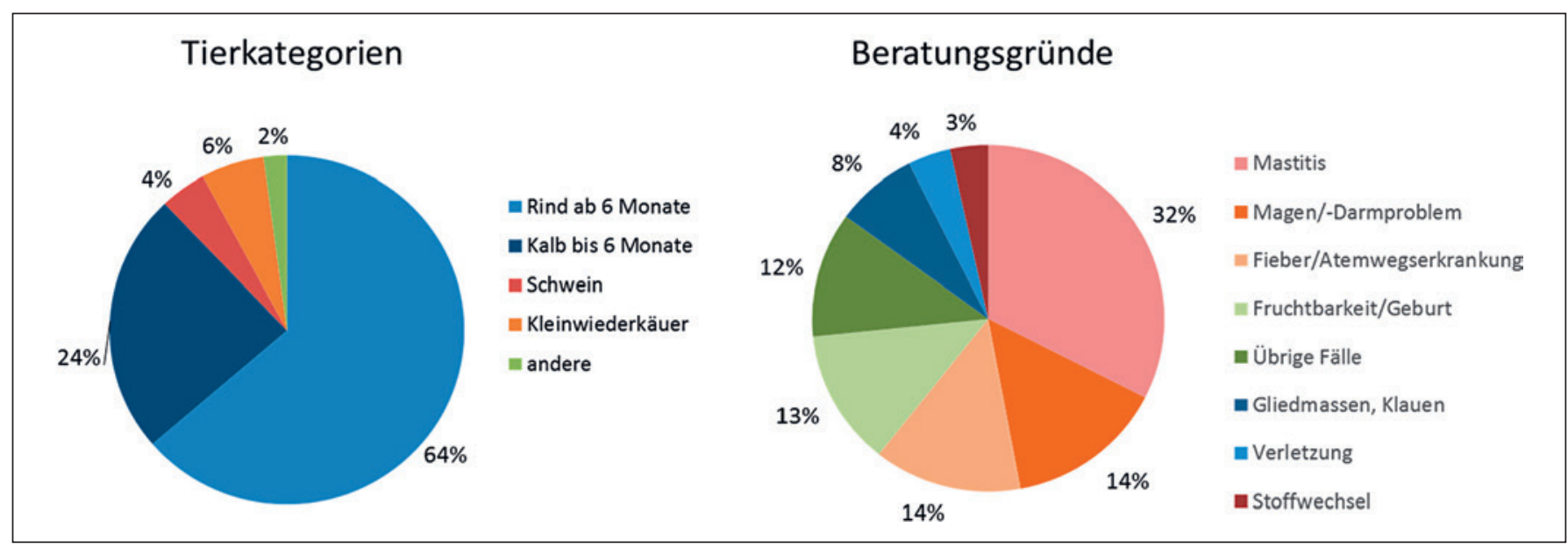

Abb. 1. Gründe für 661 Beratungsanfragen an Kometian sowie die damit verbundenen Tierkategorien, erfasst im Rahmen der Qualitätssicherung von Kometian.

Abb. 2. Krankheitsverlauf von beratenen Fällen $(n=661)$ nach Angaben der Land-

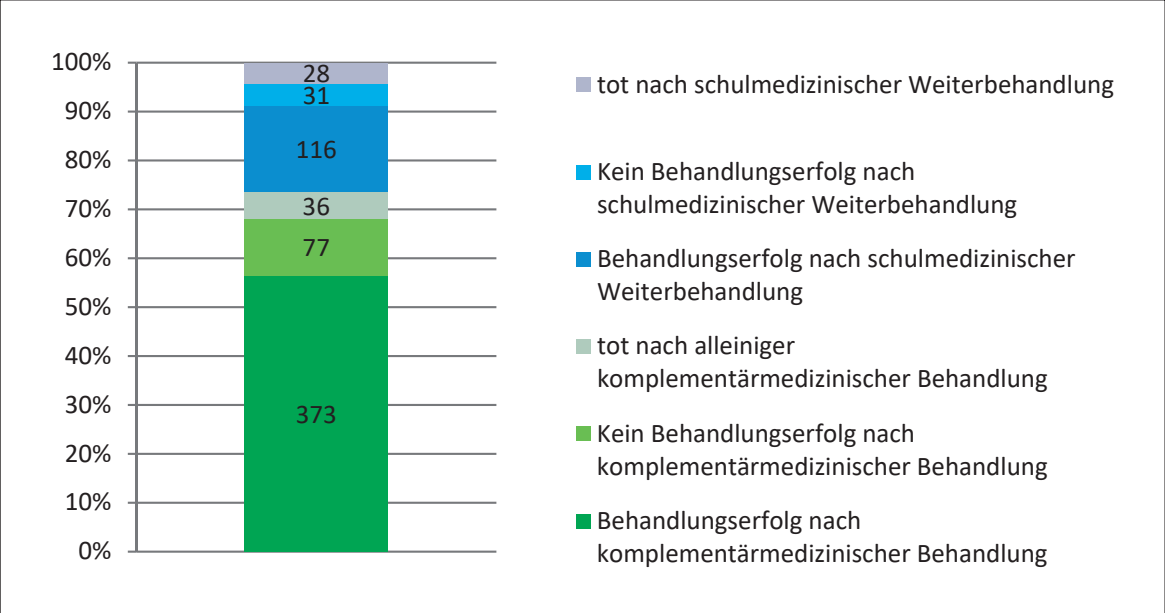
wirte.

zum grossen Teil schon erfolglos schulmedizinisch vorbehandelt waren (Kometian als "letzte Hoffnung").

In 175 Fällen (26\%) wurde das Tier zwischen Kometianberatung und telefonischer Nachfrage weiter schulmedizinisch behandelt. Davon wurden 116 als "Behandlungserfolg" gewertet (Abb. 2).

\section{Antibiotische Behandlungen auf Bestandesebene}

Der Betriebsmittelwert antibiotischer Behandlungen pro 100 Nutztiere und Jahr belief sich auf durchschnittlich 27 Behandlungen im Vorprojektjahr (Jahr 0) und reduzierte sich um einen Drittel auf 18 Behandlungen im Mittel im ersten Projektjahr (Jahr 1). Diese Differenz war hoch signifikant $(p<0,001)$. Die höchste Behandlungsinzidenz mit ebenfalls signifikanter Reduktion des Antibiotikaeinsatzes war in der Tierkategorie Milchkühe zu verzeichnen (42 Behandlungen/100 Kühe im Jahr 0 und 28 im Jahr 1, inklusive antibiotische Trockensteller) ( $p=$ 0,007). In Schweinebeständen reduzierte sich der Antibi- otikaeinsatz um mehr als 75\% ( $p=0,066)$, was einer tendenziellen Reduktion entspricht. In den Tierkategorien Jungrinder sowie Kleinwiederkäuer konnten keine signifikanten Reduktionen erreicht werden; die Behandlungsinzidenz blieb bei diesen Tierkategorien in den beiden Jahren konstant (Tab. 3).

Von den 46 Betrieben, von welchen die Behandlungsjournale ausgewertet werden konnten, nutzten zwei Drittel der Landwirte im ersten Projektjahr mindestens einmal das Beratungstelefon, jedoch nur knapp ein Drittel ersuchte 5-mal oder häufiger um die Beratung.

\section{Diskussion}

Einzeltierberatung - gesundheitliche Entwicklung der beratenen Fälle

Das Konzept von Kometian, die überwiegend telefonische komplementärmedizinische Beratung, wird kon- 
Tabelle 3. Antibiotische Behandlungen (AB) pro Tier und Jahr im Bestand: Vergleich Jahr 0/Jahr 1

\begin{tabular}{lllr}
\hline & $\begin{array}{l}\text { Mittelwert } \\
\text { AB } \\
\text { pro 100 Tiere Jahr 0 }\end{array}$ & $\begin{array}{l}\text { Mittelwert } \\
\text { AB } \\
\text { pro 100 Tiere Jahr 1 }\end{array}$ & $p$-Wert \\
\hline Alle Tiere $(n=46$ Betriebe) & $27 \pm 26$ & $18 \pm 19$ & $<0,001$ \\
Milchkühe $(n=28$ Bestände) & $42 \pm 43$ & $28 \pm 33$ & 0,007 \\
Mutterkühe $(n=15$ Bestände) & $21 \pm 15$ & $17 \pm 10$ & 0,779 \\
Jungrinder/Kälber $(n=40$ Bestände) & $19 \pm 30$ & $19 \pm 44$ & 0,449 \\
Schweine $(n=13$ Bestände) & $13 \pm 20$ & $3 \pm 10$ & 0,066 \\
Kleinwiederkäuer $(n=16$ Bestände $)$ & $3 \pm 10$ & $3 \pm 9$ & 1 \\
\hline
\end{tabular}

trovers diskutiert, da der jeweilige Berater meist weder das Tier noch den Betrieb kennt. Eine gute Mitarbeit und Tierbeobachtung des Landwirts sind für die Früherkennung von Krankheiten und eine erfolgreiche homöopathische Beratung sehr wichtig. Daher wird die Mitarbeit des Tierhalters ebenfalls erfasst und Schulungen zur Tierbeobachtung angeboten. Aktuell gibt es in der Schweiz nur wenige Tierärztinnen und Tierärzte, welche die komplementärmedizinische Therapie von Nutztieren anbieten. Die telefonische Beratung erleichterte potentiell allen deutschsprachigen Landwirtinnen und Landwirten in der Schweiz den Zugang zur Komplementärmedizin. Diese Art der Beratung kann und möchte die tierärztliche Untersuchung am Tier nicht ersetzen, kann aber den Landwirt unterstützen und motivieren, komplementärmedizinische Behandlungsmöglichkeiten anzuwenden. Die kontinuierlich steigende Zahl an Kometian-Mitgliedsbetrieben dokumentiert diesen Bedarf. Im Gegensatz zur Veterinärmedizin ist es im Bereich der Humanmedizin durchaus üblich, per Telefon medizinische Beratung anzubieten und in Anspruch zu nehmen [17, 18]. Dies wird sogar von den Schweizer Krankenkassen mit Prämienvergünstigungen gefördert [19]. Im Gegensatz $\mathrm{zu}$ Kometian, welches eine reine Beratungsleistung anbietet, können Ärztinnen und Ärzte in der Schweiz im Bereich der Telemedizin auch Rezepte für Medikamente für die ratsuchende Person selbst oder auch deren Kinder ausstellen [20-22].

Nicht unerwähnt bleiben sollte, dass davon auszugehen ist, dass der Grossteil der in der Schweiz für Nutztiere durchgeführten komplementärmedizinischen Therapien ohne Rücksprache mit Experten direkt durch Tierhalter erfolgen. Diese Form der "Selbstmedikation" ist auch aus anderen Ländern Europas bekannt [23] und nicht nur für Tierhalter beschrieben. Auch aus der Humanmedizin ist bekannt, dass Eltern beispielsweise ihre Kinder eigenverantwortlich mit Over-the-counter-Medikamenten therapieren, zu denen ja auch der Grossteil der komplementärmedizinisch eingesetzten Medikamente gehört [24]. Der Einbezug einer telefonischen Beratung, wie Kometian sie anbietet, könnte gegenüber der ausschliesslichen Selbst- medikation durch Landwirte ein Vorteil sein, nicht zuletzt auch hinsichtlich der Abwägung, ob der Hoftierarzt zugezogen werden soll.

Die telefonische Nachfrage hinsichtlich der Veränderung des Gesundheitszustands des Einzeltieres nach einer Beratung kann nicht mit einer klinisch-tierärztlichen Untersuchung gleichgesetzt werden. Das angewandte Instrument der Qualitätssicherung geht dennoch weit über das in der tierärztlichen Praxis Übliche hinaus. Die Kriterien, nach denen die Aussage des Landwirtes als Behandlungserfolg gewertet wurde, waren streng. Nur Tiere, welche vom Landwirt als nahezu oder ganz gesund angesehen wurden und bis zum Zeitpunkt der Nachkontrolle keine weitere schulmedizinische Behandlung benötigten, um weiter genutzt werden zu können, wurden in diese Kategorien eingeteilt.

Rund drei Viertel der beratenen Fälle zeigten, nach Angaben der Landwirte, einen positiven Verlauf (57\% nach alleiniger komplementärmedizinischer Behandlung, $16 \%$ nach schulmedizinischer Weiterbehandlung). In 9,7\% der Fälle konnte der Tod des Tieres nicht verhindert werden, was in einem mit internationalen Studien vergleichbaren Rahmen liegt. So beschreibt eine Schweizer Studie eine Mortalität von 4,1\% bei Mastkälbern unter Praxisbedingungen [25], in einer schwedischen Studie erkrankten in den ersten 3 Lebensmonaten 708 von 3'081 beobachteten weiblichen Milchviehkälbern. 90 Kälber verendeten in diesem Zeitraum, was einer Letalität von 13\% entspricht [26]. Die häufigsten Todesursachen waren jeweils Durchfälle und Atemwegserkrankungen. Die Mortalität bei laktierenden Milchkühen innerhalb eines Jahres liegt zwischen 2,1 und $8,1 \%$ [27-29].

Vergleichszahlen zur Gesundheitsentwicklung oder Letalität von Nutztieren nach praxisüblicher Behandlung fehlen, sowohl aus der Schul- als auch aus der Komplementärmedizin. Zusammenfassend und basierend auf der telefonischen Nachfrage scheint es durchaus eine weiterzuverfolgende Methode, eine komplementärmedizinische Behandlung als Ersttherapie zu wählen und bei Bedarf die Therapie später anzupassen. 
Tabelle 4. Antibiotikaeinsatz bei Milchkühen: Vergleichszahlen aus der Schweiz

\begin{tabular}{lllll}
\hline & $\begin{array}{l}\text { Erhebung } \\
\text { Kometian 2015 } \\
\text { Jahr 0 }(n=28)\end{array}$ & $\begin{array}{l}\text { Erhebung } \\
\text { Kometian 2015 } \\
\text { Jahr 1 }(n=28)\end{array}$ & $\begin{array}{l}\text { Erhebung } \\
\text { ALP 2003/2004 } \\
{[32](n=76)}\end{array}$ & $\begin{array}{l}\text { Erhebung } \\
\text { Bio Suisse 2010 } \\
{[31](n=51)}\end{array}$ \\
\hline $\begin{array}{l}\text { Anzahl antibiotische Behandlungen/ } \\
\text { 100 Kühe und Jahr }\end{array}$ & $42 \pm 43$ & $28 \pm 33$ & $83 \pm 51$ & 51 \\
\hline
\end{tabular}

Antibiotische Behandlungen auf Bestandesebene

Obwohl lediglich ein Drittel der Landwirte, welche die Behandlungsjournale zur Verfügung stellten, im ersten Projektjahr das Beratungstelefon nutzten, konnte ein deutlicher Rückgang des Antibiotikaeinsatzes erreicht werden. Die komplementärmedizinische Beratung scheint also nicht allein der Grund der Antibiotikareduktion auf den Betrieben zu sein. Denkbar wäre, dass die Projektteilnahme als solches bereits eine Motivation für die Reduktion von Antibiotika dargestellt hat. Nahezu alle teilnehmenden Landwirte gaben an, schon vor Projektbeginn Erfahrung mit der Komplementärmedizin gesammelt zu haben, und es erscheint möglich, dass die Tierhalter mit der Sicherheit, bei Bedarf rund um die Uhr auf eine komplementärmedizinische Beratung zurückgreifen zu können, ihre Tiere vermehrt selbst komplementärmedizinisch behandelt haben. Unterstützt wird diese mögliche Erklärung durch eine Untersuchung von Stevens et al. [30] auf belgischen Milchviehbetrieben. Hierbei konnte festgestellt werden, dass Landwirte, welche homöopathische Medikamente verwendeten, insgesamt weniger Antibiotika einsetzten. Ob eine generelle Zunahme komplementärmedizinischer Behandlungen, welche der Landwirt in Eigenregie durchführte, zu diesem Ergebnis führen konnte, bleibt jedoch unklar, da komplementärmedizinische Behandlungen nur bedingt der Aufzeichnungspflicht unterworfen sind und somit retrospektiv nicht verlässlich erhoben werden können.

Mit 42 Behandlungen auf 100 Kühe im Vorprojektjahr war auf Milchviehbetrieben das grösste Reduktionspotential vorhanden, obwohl diese Ausgangssituation schon deutlich unter den Angaben der durchschnittlichen Behandlungsinzidenz schweizerischer Milchviehbetriebe liegt [31, 32] (Tab. 4). Vergleichbar hingegen ist diese Ausgangssituation mit den Studien von Ivemeyer et al. von 2008 und 2012 [6,33] zur Antibiotikareduktion beim Milchvieh. Wie in diesen Studien schon gezeigt, konnten die Betriebe im Projektjahr 1 von Kometian den Antibiotikaeinsatz noch um weitere 30\% reduzieren (Tab. 4). Diese Reduktion wurde allerdings mit erheblich geringerem Aufwand erreicht, als dies bei Ivemeyer et al. [33], Vaarst et al. [34] oder Tschopp et al. [35] beschrieben wurde.

Ein komplementärmedizinisches

Beratungssystem für Nutztiere
In einer Untersuchung auf Betrieben von Schweizer Schweineproduzenten konnten Malik et al. [36] aufzeigen, dass die persönliche Einstellung des Landwirts erhebliche Effekte auf den betrieblichen Antibiotikaeinsatz hat. Dass die Einstellung und das Verhalten des Landwirtes Einfluss auf die Eutergesundheit haben, zeigten auch Studien von Jansen et al. [37], Ivemeyer et al. [38] und Schewe et al. [39]. Tschopp et al. [35] konnten zeigen, dass die Auseinandersetzung des Landwirts selbst mit Tiergesundheitsthemen zu einer Reduktion von antibiotischen Mastitisbehandlungen führte, während punktuelle Inanspruchnahme von Beratung oder regelmässige Betreuung durch den Hoftierarzt keinen Einfluss zeigten oder sogar zu einer Erhöhung der eingesetzten Antibiotikamenge führten.

In der Schweiz sind zurzeit keine repräsentativen und detaillierten Aufzeichnungen über den Einsatz von Antibiotika beim Nutztier vorhanden, es sind allein Daten zum Antibiotikavertrieb erhältlich [1]. Seit 2009 ist eine stetige Abnahme der verkauften Menge Antibiotika in der Tiermedizin zu verzeichnen [1]. Im Jahr 2014 beispielsweise wurden $8 \%$ weniger Antibiotika in der Veterinärmedizin verkauft als im Vorjahr. Wenngleich diese Angaben auf reinen Verkaufszahlen beruhen und keine Aussage über die Behandlungsinzidenz oder über die Tierarten, bei welchen die Medikamente eingesetzt wurden, zulassen, scheinen die Betriebe von Kometian den Antibiotikaeinsatz innerhalb eines Jahres jedoch dennoch deutlich stärker reduziert zu haben. Eine schweizweite Erfassung dieser Daten könnte nicht zuletzt auch dazu beitragen, aussagekräftige Vergleichszahlen für Projekte zu erhalten, welche zum Ziel haben, den Antibiotikaeinsatz zu verringern. Beispielsweise ist aus den Niederlanden bekannt, dass über alle Nutztierarten gleichermassen der Antibiotikaeinsatz landesweit stark reduziert werden konnte [40]. Solche generellen Trends sind für Studien, welche Veränderungen im Medikamenteneinsatz als Zielvariable haben, generell miteinzubeziehen und zu diskutieren.

Seit Anfang 2016 ist Kometian ein vom Bundesamt für Landwirtschaft gefördertes Ressourcenprojekt. In diesem Rahmen werden in den nächsten Jahren weitere Untersuchungen durchgeführt, um die Entwicklung von Tierge- 
sundheit und Antibiotikaeinsatz auf komplementärmedizinisch beratenen Betrieben längerfristig zu untersuchen.

\section{Schlussfolgerung}

Die telefonische komplementärmedizinische Beratung von Landwirten zur Behandlung von Einzeltieren scheint zumindest aus Sicht der Landwirte einen positiven Einfluss auf die Entwicklung des Gesundheitszustandes des Einzeltieres zu haben. Gesamtbetrieblich führt die Teilnahme an einem solchen Beratungsprojekt zur deutlichen Reduktion des Antibiotikaeinsatzes, insbesondere beim Milchvieh. Für die weiteren Tierarten und Produktionsrichtungen müssten mehr und grössere Bestände analysiert werden, um genauere Aussagen ma- chen zu können. Zudem sollte zukünftig zumindest stichprobenartig durch Betriebsbesuche die Einschätzung der Entwicklung der Tiergesundheit durch den Landwirt mit direkt am Tier erhobenen Daten abgeglichen werden.

\section{Statement of Ethics}

Die Beraterinnen und Berater von "Kometian" gaben ihre Behandlungsempfehlungen nach bestem Wissen und Gewissen ab, gemäss den Richtlinien der "Good Veterinary Practice". In schwierigen Fällen wurden die Tierhalter angewiesen ihren Hoftierarzt beizuziehen, um das Tier vor Ort abklären und behandeln zu lassen.

\section{Disclosure Statement}

Die Autoren bestätigen, dass keine Interessenskonflikte bestehen.

\section{Literatur}

1 Bundesamt für Lebensmittelsicherheit und Veterinärwesen (BLV). ARCH-Vet Kurzversion: Bericht über den Vertrieb von Antibiotika in der Veterinärmedizin in der Schweiz. 2016 [zitiert 14. März 2018]. https://www.blv. admin.ch/blv/de/home/tiere/publikationenund-forschung/statistiken-berichte-tiere. html.

2 Binh CT, Heuer H, Kaupenjohann M, Smalla K. Piggery manure used for soil fertilization is a reservoir for transferable antibiotic resistance plasmids. FEMS Microbiol Ecol. 2008 Oct;66(1):25-37.

3 Johnson TA, Stedtfeld RD, Wang Q, Cole JR, Hashsham SA, Looft T, et al. Clusters of Antibiotic Resistance Genes Enriched Together Stay Together in Swine Agriculture. MBio. 2016 Apr;7(2):e02214-5.

4 Bonde M, Sorensen JT. Herd health management in organic pig production using a quality assurance system based on Hazard Analysis and Critical Control Points. NJAS Wagening J Life Sci. 2004;52(2):133-43.

5 Ivemeyer S, Walkenhorst M, Heil F, Notz C, Maeschli A, Butler G, et al. Management factors affecting udder health and effects of a one year extension program in organic dairy herds. Animal. 2009 Nov;3(11):1596-604.

6 Ivemeyer S, Smolders G, Brinkmann J, Gratzer E, Hansen B, Henriksen BI, et al. Impact of animal health and welfare planning on medicine use, herd health and production in European organic dairy farms. Livest Sci. 2012;145(1-3):63-72.

7 Schweizerische Eidgenossenschaft. Bundesverfassung Art. 18a https://www.admin.ch/ opc/de/classified-compilation/19995395/index.html

8 Mayer M, Vogl CR, Amorena M, Hamburger M, Walkenhorst M. Treatment of organic livestock with medicinal plants: a systematic review of European ethnoveterinary research. Forsch Komplementmed. 2014;21(6):375-86.
9 Walkenhorst M, Vogl CR, Vogl-Lukasser B, Vollstedt S, Brendieck-Worm C, Ivemeyer S, et al. Zwischen Empirie und Evidenz: (Re)aktivierung der Veterinärphytotherapie. Forsch Komplement Med. 2014;21(1 Suppl 1):35-42.

10 Bischoff T, Vogl CR., Ivemeyer S, Klarer F, Meier B, Hamburger M, Walkenhorst M. Plant and natural product based homemade remedies manufactured and used by farmers of six central Swiss cantons to treat livestock. Livest Sci. 2016 Jul;189:110-25.

11 Hovi M. Alternative therapy use on UK organic farms: constraints and pitfalls. Proceedings of the Fifth NAHWOA Workshop. Rødding, Denmark - November 11-13, 2001; p. 7-13.

12 Hektoen L. Review of the current involvement of homeopathy in veterinary practice and research. Vet Rec. 2005 Aug;157(8):2249.

13 Kometian. http://www.kometian.ch [zitiert 14. März 2018].

14 Camvet. http://www.camvet.ch [zitiert 14. März 2018].

15 Tierheilpraktikerverband. http://www.tierheilpraktikerverband.ch [zitiert 14. März 2018].

16 Homöopathieverband Schweiz. http://www. hvs.ch [zitiert 14. März 2018].

17 Medgate. http://www.medgate.ch [zitiert 14. März 2018].

18 Medi24. http://www.medi24.ch [zitiert 14. März 2018].

19 Comparis.ch. http://krankenkasse.ch/ telmed-modell/ [zitiert 14. März 2018].

20 Visana. https://www.visana.ch/de/privatkunden/services/medi24 [zitiert 14. März 2018].

21 Medgate. https://www.medgate.ch/de-ch/ telemedicinecenter\#1744622-rezeptierung [zitiert 14. März 2018].

22 OEKK. https://www.oekk.ch/de/privatkunden/angebote/sparen-und-vorteile/vorteile/ aerztlicher-rat $/ \#$ accid $=37$ B6B28D-ECE443BE-B6E7-C79827B8F55C [zitiert 14. März 2018].
23 Doehring C, Sundrum A. Efficacy of homeopathy in livestock according to peer-reviewed publications from 1981 to 2014 . Vet Rec. 2016 Dec;179(24):628.

24 Siponen S, Ahonen R, Kiviniemi V, HämeenAnttila K. Association between parental attitudes and self-medication of their children. Int J Clin Pharm. 2013 Feb;35(1):113-20.

25 Lava M, Schüpbach-Regula G, Steiner A, Meylan M. Antimicrobial drug use and risk factors associated with treatment incidence and mortality in Swiss veal calves reared under improved welfare conditions. Prev Vet Med. 2016 Apr;126:121-30.

26 Svensson C, Lundborg K, Emanuelson U, Olsson SO. Morbidity in Swedish dairy calves from birth to 90 days of age and individual calf-level risk factors for infectious diseases. Prev Vet Med. 2003 May;58(3-4):179-97.

27 Thomsen PT, Kjeldsen AM, Sørensen JT, Houe H. Mortality (including euthanasia) among Danish dairy cows (1990-2001). Prev Vet Med. 2004 Jan;62(1):19-33.

28 Hertl JA, Schukken YH, Bar D, Bennett GJ, González RN, Rauch BJ, et al. The effect of recurrent episodes of clinical mastitis caused by gram-positive and gram-negative bacteria and other organisms on mortality and culling in Holstein dairy cows. J Dairy Sci. 2011 Oct; 94(10):4863-77.

29 Shahid MQ, Reneau JK, Chester-Jones H, Chebel RC, Endres MI. Cow- and herd-level risk factors for on-farm mortality in Midwest US dairy herds. J Dairy Sci. 2015 Jul;98(7): 4401-13.

30 Stevens M, Piepers S, De Vliegher S. Mastitis prevention and control practices and mastitis treatment strategies associated with the consumption of (critically important) antimicrobials on dairy herds in Flanders, Belgium. J Dairy Sci. 2016 Apr;99(4):2896-903.

31 Seidel K. Erfassung des Antibiotikaeinsatzes in der biologischen Milchproduktion. Schlussbericht einer Erhebung der Bio Suisse 2010. 
32 Schaeren W. Antibiotikaverbrauch 2003 und 2004 in der Milchproduktion. Agrarforschung. 2006;13(6):234-9.

33 Ivemeyer S, Maeschli A, Walkenhorst M, Klocke P, Heil F, Oser S, et al. Auswirkungen einer zweijährigen Bestandesbetreuung von Milchviehbeständen hinsichtlich Eutergesundheit, Antibiotikaeinsatz und Nutzungsdauer. Schweiz Arch Tierheilkd. 2008 Oct; 150(10):499-505.

34 Vaarst M, Nissen TB, Østergaard S, Klaas IC, Bennedsgaard TW, Christensen J. Danish stable schools for experiential common learning in groups of organic dairy farmers. J Dairy Sci. 2007 May;90(5):2543-54
35 Tschopp A, Reist M, Kaufmann T, Bodmer M, Kretzschmar L, Heiniger D, et al. A multiarm randomized field trial evaluating strategies for udder health improvement in Swiss dairy herds. J Dairy Sci. 2015 Feb;98(2):84060.

36 Malik J, Kaufmann G, Hirsiger P, Kümmerlen D, Arnold C, Spring P, et al. Einfluss der persönlichen Einstellung des Betriebsleiters auf den Antibiotika-Verbrauch in der Schweineproduktion. Schweiz Arch Tierheilkd. 2015 Dec;157(12):675-81.

37 Jansen J, van den Borne BH, Renes RJ, van Schaik G, Lam TJ, Leeuwis C. Explaining mastitis incidence in Dutch dairy farming: the influence of farmers' attitudes and behaviour. Prev Vet Med. 2009 Nov;92(3):210-23.

38 Ivemeyer S, Knierim U, Waiblinger S. Effect of human-animal relationship and management on udder health in Swiss dairy herds. J Dairy Sci. 2011 Dec;94(12):5890-902.
39 Schewe RL, Kayitsinga J, Contreras GA, Odom C, Coats WA, Durst P, et al. Herd management and social variables associated with bulk tank somatic cell count in dairy herds in the eastern United States. J Dairy Sci. 2015 Nov;98(11):7650-65.

40 Ministry of Economic Affairs. Reduced and Responsible Policy on the use of antibiotics in food-producing animals in the Netherlands 2004; https://www.government.nl/binaries/government/documents/leaflets/2014/02/28/reduced-and-responsibleuse-of-antibiotics-in-food-producing-ani$\mathrm{m}$ a l s - i n - the - n e the r la nd s/ use-of-antibiotics-in-food-producing-animals-in-the-netherlands.pdf. 